\title{
Distributed Centroid Estimation from Noisy Relative Measurements
}

\author{
Rosario Aragues ${ }^{\mathrm{a}, *}$, Luca Carlone ${ }^{\mathrm{b}, \mathrm{d}}$, Carlos Sagues $^{\mathrm{a}}$, Giuseppe Calafiore ${ }^{\mathrm{c}, \mathrm{d}}$ \\ ${ }^{a}$ Departamento de Informática e Ingeniería de Sistemas, Instituto de Investigación en \\ Ingeniería de Aragón, Universidad de Zaragoza, María de Luna 1, 50018 Zaragoza, Spain \\ ${ }^{b}$ CSPP, Laboratorio di Meccatronica \\ ${ }^{c}$ Dipartimento di Automatica e Informatica \\ ${ }^{d}$ Politecnico di Torino, Corso Duca degli Abruzzi 24, 10129 Torino, Italy
}

\begin{abstract}
We propose an anchorless distributed technique for estimating the centroid of a network of agents from noisy relative measurements. The positions of the agents are then obtained relative to the estimated centroid. The usual approach to multi-agent localization assumes instead that one anchor agent exists in the network, and the other agents positions are estimated with respect to the anchor. We show that our centroid-based algorithm converges to the optimal solution, and that such a centroid-based representation produces results that are more accurate than anchor-based ones, irrespective of the selected anchor.
\end{abstract}

Keywords: Distributed systems, Sensor networks, Noisy relative measurements, Multi-agent localization, Distributed linear estimation

\section{INTRODUCTION}

Several multi-agent tasks require the knowledge of the agents' positions in a common reference frame, e.g, for combining information taken by a camera network [1], or for algorithms explicitly involving the communication of positions, or that specifically require the knowledge of the centroid [2]. In these

\footnotetext{
*Corresponding author. Ph.: (+34)976761949, Fax: (+34)976761914

Email addresses: raragues@unizar.es (Rosario Aragues), luca.carlone@polito.it

(Luca Carlone), csagues@unizar.es (Carlos Sagues), giuseppe.calafiore@polito.it

(Giuseppe Calafiore)
}

Preprint submitted to Systems \& Control Letters

February 13, 2012 
cases, the common frame is a necessary tool for combining spatial information acquired by the different agents. Therefore, it is interesting to place the origin or the global frame, in our case the centroid of the positions, where it provides more accurate results. Typically, agents start at unknown locations, they do not share any common frame, and they can only measure the relative positions of nearby agents. Here, we address the localization problem, which consists of combining these relative measurements to build an estimate of the agents' positions in a common frame. We focus on the position estimation problem and assume that all the local frames have a common orientation, because the agents have a sensor (e.g., a compass) that gives them their global orientation, or because they have previously executed an attitude synchronization [3] or a motion coordination [4] strategy to align their orientations.

Several localization algorithms rely on range-only $[5,6]$, or bearing-only [7] relative measurements of positions. Alternatively, each agent can locally combine its observations and build an estimate of the relative full-position of its neighbors using e.g., the approach described in $[8,9]$ for $3 \mathrm{D}$ scenarios. When full-position measurements are available, the localization problem becomes linear and can thus be solved by using linear optimization methods [10-12]. We address this latter scenario, where agents measure the relative $p$-dimensional full-position of their neighbors, being the measurements corrupted with noise. There exist works that compute not only the agents' positions but also their orientations [13], and that track the agents' poses [14]. A related problem is formation control $[4,15-17]$, where agents move to positions satisfying a set of desired inter-agent restrictions (range-only, bearing-only, full-positions, or relative poses). Although some works discuss the effects of measurement noises in the final result [4], formation algorithms usually assume that both, the measurements and the inter-agent restrictions are noise free [15-17].

Both, formation control and localization problems can be solved up to a rotation and a translation. This ambiguity disappears when the positions of a subset of anchor agents is given in some absolute reference frame. The density and placement of anchors has an important effect on the accuracy of the solu- 
tions for the bearing-only and range-only cases $[5,7]$. In the full-position case, a single anchor is enough. Its placement influences the accuracy of the final results, and the estimation errors at the agents are usually analyzed as a function of their distances to the anchor [18]. However, it is common to assume that the first agent is the anchor placed at the origin of the common reference frame and make the other agents compute their positions relative to the anchor. Here, instead, we discuss the use of the centroid of the team as the common frame. We show that the centroid-based algorithm produces more accurate results than any anchor selection.

The centroid of the team or a weighted average of the agents' positions has been previously used as the common frame in the contexts of localization from noise-free measurements [13] or formation control [17]. The rendezvous and formation control strategies in [15] are shown to conserve the centroid of the initial configuration. These previous works have in common that the measurements are noise-free, whereby the centroid can be easily obtained. In this paper we define the centroid in the presence of noisy measurements. To our knowledge, the centroid has not been applied to the problem of localization from noisy relative position measurements yet. As we show next, using the centroid as the reference frame for the multi-agent localization problem does not introduce additional complexity in the algorithm and it provides more accurate results.

The contributions of this paper are: (i) We propose a novel strategy where the agents compute their states, in presence of noisy measurements, relative to the centroid in a distributed fashion. (ii) We formally prove that this strategy leads the system to the same optimal centroid solution that would be obtained by a centralized system. (iii) An additional contribution refers to the computation of the anchor-based states with the Jacobi algorithm [10, 19]. We provide a proof for convergence (Theorem 2) under independent measurement noises with general covariance matrices, not necessarily diagonal or equal.

The paper is organized as follows. Section 2 presents the multi-agent estimation problem. Section 3 discusses the estimation relative to an anchor. Section 4 presents the centroid-based estimation problem and Section 5 presents our dis- 
tributed solution. Section 6 shows the performance of the algorithm.

\section{PRELIMINARIES}

Consider a set of $n \in \mathbb{N}$ agents. Each agent $i \in\{1, \ldots, n\}$ has a $p$-dimensional state $\mathbf{x}_{i} \in \mathbb{R}^{p}$ and it observes the states of a subset of the agents relative to its own state, $\mathbf{x}_{j}-\mathbf{x}_{i}$. These states can be, for instance, positions in cartesian coordinates, orientations, speeds, accelerations, or current times. There are $m$ relative measurements. This information is represented by a directed graph $\mathcal{G}=(\mathcal{V}, \mathcal{E})$, where the nodes $\mathcal{V}=\{1, \ldots, n\}$ are the agents, and $\mathcal{E}$ contains the $m$ relative measurements, $|\mathcal{E}|=m$. There is an edge $e=(i, j) \in \mathcal{E}$ from $i$ to $j$ if agent $i$ has a noisy relative measurement $\mathbf{z}_{e} \in \mathbb{R}^{p}$ of the state of agent $j$, $\mathbf{z}_{e}=\mathbf{x}_{j}-\mathbf{x}_{i}+\mathbf{v}_{e}$, where $\mathbf{v}_{e} \sim N\left(\mathbf{0}_{p \times p}, \Sigma_{\mathbf{z}_{e}}\right)$ is a Gaussian additive noise. We let $\mathbf{z}, \mathbf{v} \in \mathbb{R}^{m p}$ and $\Sigma_{\mathbf{z}} \in \mathbb{R}^{m p \times m p}$ contain the information of the $m$ measurements ${ }^{1}$,

$$
\mathbf{z}=\left(\mathbf{z}_{1}^{T}, \ldots, \mathbf{z}_{m}^{T}\right)^{T}, \quad \mathbf{v}=\left(\mathbf{v}_{1}^{T}, \ldots, \mathbf{v}_{m}^{T}\right)^{T}, \quad \Sigma_{\mathbf{z}}=\operatorname{blkDiag}\left(\Sigma_{\mathbf{z}_{1}}, \ldots, \Sigma_{\mathbf{z}_{m}}\right) .
$$

Each agent $i$ communicates with both, its in and out neighbors $\mathcal{N}_{i}$ in $\mathcal{G}$, so that the communication graph $\mathcal{G}_{\text {com }}=\left(\mathcal{V}, \mathcal{E}_{\text {com }}\right)$ is the undirected version of $\mathcal{G}$,

$$
\mathcal{E}_{\text {com }}=\{\{i, j\} \mid(i, j) \in \mathcal{E} \text { or }(j, i) \in \mathcal{E}\}, \quad \mathcal{N}_{i}=\left\{j \mid\{i, j\} \in \mathcal{E}_{\text {com }}\right\}
$$

We assume that $\mathcal{G}$ is weakly connected; equivalently, $\mathcal{G}_{\text {com }}$ is connected.

The estimation from relative measurements problem consists of estimating the states of the $n$ agents from the measurements z. Any solution is determined only up to an additive constant. Conventionally [10] one agent $a \in \mathcal{V}$, e.g., the first one $a=1$, is established as an anchor with state $\hat{\mathbf{x}}_{a}^{a}=\mathbf{0}_{p}$. We call such approaches anchor-based and add the superscript $a$ to their associated variables. Vector $\mathbf{z}$ is a measurement of the true relative states $\mathbf{x}_{\mathcal{V}^{a}}^{a} \in \mathbb{R}^{(n-1) p}$ of the nonanchor agents $\mathcal{V}^{a}=\mathcal{V} \backslash\{a\}$ relative to $a, \mathbf{z}=\left(\mathcal{A}^{a} \otimes \mathbf{I}_{p}\right)^{T} \mathbf{x}_{\mathcal{V}^{a}}$, where the noises

\footnotetext{
${ }^{1} A=\operatorname{blkDiag}\left(B_{1}, \ldots, B_{r}\right)$ returns a matrix $A$ defined by blocks with $A_{i i}=B_{i}$ and $A_{i j}=\mathbf{0}$ for $i \neq j$.
} 
satisfy $\mathbf{v} \sim N\left(\mathbf{0}, \Sigma_{\mathbf{z}}\right)$, and where $\mathcal{A}^{a} \in \mathbb{R}^{(n-1) \times m}$ is the incidence matrix of $\mathcal{G}$ but without the row associated to the anchor,

$$
\mathcal{A}_{i, e}^{a}=-1 \text { if } e=(i, j), \quad \mathcal{A}_{i, e}^{a}=1 \text { if } e=(j, i), \text { and } \quad \mathcal{A}_{i, e}^{a}=0 \text { otherwise. }
$$

Thus, the Best Linear Unbiased Estimator $\hat{\mathbf{x}}_{\mathcal{V}^{a}} \in \mathbb{R}^{(n-1) p}$ of the states of the non-anchor agents $\mathcal{V}^{a}$ relative to the anchor $a$ are given by the following Weighted Least Squares approximate solution [10],

$$
\hat{\mathbf{x}}_{\mathcal{V}^{a}}^{a}=\Sigma_{\hat{\mathbf{x}}_{\mathcal{V}^{a}}^{a}}\left(\mathcal{A}^{a} \otimes \mathbf{I}_{p}\right) \Sigma_{\mathbf{z}}^{-1} \mathbf{z}, \quad \Sigma_{\hat{\mathbf{x}}_{\mathcal{V}^{a}}^{a}}=\left(\left(\mathcal{A}^{a} \otimes \mathbf{I}_{p}\right) \Sigma_{\mathbf{z}}^{-1}\left(\mathcal{A}^{a} \otimes \mathbf{I}_{p}\right)^{T}\right)^{-1} .
$$

From now on, both $\hat{\mathbf{x}}_{\mathcal{V}}^{a}=\left(\mathbf{0}_{p}^{T},\left(\hat{\mathbf{x}}_{\mathcal{V}^{a}}^{a}\right)^{T}\right)^{T}$ and $\Sigma_{\hat{\mathbf{x}}_{\mathcal{V}}^{a}}=\operatorname{blkDiag}\left(\mathbf{0}_{p \times p}, \Sigma_{\hat{\mathbf{x}}_{\mathcal{V}^{a}}^{a}}\right)$, include the estimated state of the anchor $a$ as well.

\section{DISTRIBUTED ESTIMATION RELATIVE TO AN ANCHOR}

We are interested in distributed strategies where each agent $i$ iteratively estimates its own state in eq. (2) through local interactions with its neighbors $\mathcal{N}_{i}$. Among the different existing methods for estimating the states $\hat{\mathbf{x}}_{\mathcal{V}}^{a}$ relative to an anchor, we use the Jacobi algorithm [10], although other distributed methods such as the Jacobi Overrelaxation [19], or the Overlapping Subgraph Estimator [20] could alternatively be applied. The approach in [11], based on the cycle structure of the graph, could be used as well, although it requires multi-hop communication.

Considering eq. (2), it can be seen that computing $\hat{\mathbf{x}}_{\mathcal{V}^{a}}^{a}$ is equivalent to finding a solution to the system $\Upsilon \hat{\mathbf{x}}_{\mathcal{V}^{a}}^{a}=\eta$, being $\eta$ and $\Upsilon$ the information vector and matrix associated to $\hat{\mathbf{x}}_{\mathcal{V}^{a}}^{a}$ and $\Sigma_{\hat{\mathbf{x}}_{\mathcal{V}}^{a}}$,

$$
\eta=\left(\mathcal{A}^{a} \otimes \mathbf{I}_{p}\right) \Sigma_{\mathbf{z}}^{-1} \mathbf{z}, \quad \Upsilon=\left(\mathcal{A}^{a} \otimes \mathbf{I}_{p}\right) \Sigma_{\mathbf{z}}^{-1}\left(\mathcal{A}^{a} \otimes \mathbf{I}_{p}\right)^{T} .
$$

This can be solved with the Jacobi method [19], which first decomposes $\Upsilon=$ $\left[\Upsilon_{i j}\right]$ into $D, N$ as follows:

$$
D=\operatorname{blkDiag}\left(\Upsilon_{22}, \ldots, \Upsilon_{n n}\right), \quad N=D-\Upsilon
$$


so that system $\Upsilon \hat{\mathbf{x}}_{\mathcal{V}^{a}}^{a}=\eta$ becomes $(D-N) \hat{\mathbf{x}}_{\mathcal{V}^{a}}^{a}=\eta$, and equivalently $\hat{\mathbf{x}}_{\mathcal{V}^{a}}^{a}=$ $D^{-1} N \hat{\mathbf{x}}_{\mathcal{V}^{a}}^{a}+D^{-1} \eta$. The Jacobi method [19] iteratively computes a solution for $\hat{\mathbf{x}}_{\mathcal{V}^{a}}^{a}=D^{-1} N \hat{\mathbf{x}}_{\mathcal{V}^{a}}^{a}+D^{-1} \eta$, by initializing a variable $\hat{\mathbf{x}}_{\mathcal{V}^{a}}^{a}(t) \in \mathbb{R}^{(n-1) p}$ with an arbitrary value $\hat{\mathbf{x}}_{\mathcal{V}^{a}}^{a}(0)$, and updating it at each step $t$ with the following rule,

$$
\hat{\mathbf{x}}_{\mathcal{V}^{a}}^{a}(t+1)=D^{-1} N \hat{\mathbf{x}}_{\mathcal{V}^{a}}^{a}(t)+D^{-1} \eta
$$

The previous variable $\hat{\mathbf{x}}_{\mathcal{V}^{a}}^{a}(t)$ converges to $\hat{\mathbf{x}}_{\mathcal{V}^{a}}^{a}$ if the Jacobi matrix $J=D^{-1} N$ has spectral radius less than or equal to one, $\rho(J)=\rho\left(D^{-1} N\right)<1$. The interest of the Jacobi method is that it can be executed in a distributed fashion when the information matrix $\Upsilon$ is compatible with the graph (if $j \notin \mathcal{N}_{i}$ then $\Upsilon_{i j}=\Upsilon_{j i}=\mathbf{0}_{p \times p}$ ), and when in addition the rows of $\Upsilon$ and of $\eta$ associated to each agent $i \in \mathcal{V}^{a}$ only depend on data which is local to agent $i$. Next, the general anchor-based estimation algorithm [10] based on the Jacobi method is presented. It allows each agent $i \in \mathcal{V}$ to iteratively estimate its own $\hat{\mathbf{x}}_{i}^{a}$ within $\hat{\mathbf{x}}_{\mathcal{V}^{a}}^{a}=\left(\hat{\mathbf{x}}_{2}^{a}, \ldots, \hat{\mathbf{x}}_{n}^{a}\right)^{T}$ in a distributed fashion.

Algorithm 1. Let each agent $i \in \mathcal{V}$ have a variable $\hat{\mathbf{x}}_{i}^{a}(t) \in \mathbb{R}^{p}$ initialized at $t=0$ with $\hat{\mathbf{x}}_{i}^{a}(0)=\mathbf{0}_{p}$. At each time step $t$, each agent $i \in \mathcal{V}$ updates $\hat{\mathbf{x}}_{i}^{a}(t)$ with

$$
\hat{\mathbf{x}}_{i}^{a}(t+1)=\sum_{j \in \mathcal{N}_{i}} M_{i} \mathcal{B}_{i j} \hat{\mathbf{x}}_{j}^{a}(t)+\sum_{e=(j, i) \in \mathcal{E}} M_{i} \Sigma_{\mathbf{z}_{e}}^{-1} \mathbf{z}_{e}-\sum_{e=(i, j) \in \mathcal{E}} M_{i} \Sigma_{\mathbf{z}_{e}}^{-1} \mathbf{z}_{e}
$$

where $M_{i}$ and $\mathcal{B}_{i j}$ are $p \times p$ matrices with $M_{i}=\mathbf{0}$ for $i=a, M_{i}=\left(\sum_{j \in \mathcal{N}_{i}} \mathcal{B}_{i j}\right)^{-1}$ for $i \neq a$, and

$$
\begin{aligned}
& \mathcal{B}_{i j}=\Sigma_{\mathbf{z}_{e}}^{-1} \text { if either } e=(i, j) \in \mathcal{E},(j, i) \notin \mathcal{E} \text {, or } e=(j, i) \in \mathcal{E},(i, j) \notin \mathcal{E} . \\
& \mathcal{B}_{i j}=\Sigma_{\mathbf{z}_{e}}^{-1}+\Sigma_{\mathbf{z}_{e^{\prime}}}^{-1}, \text { if both } e=(i, j) \text { and } e^{\prime}=(j, i) \in \mathcal{E} .
\end{aligned}
$$

The convergence of this estimation algorithm has been proved [10, Theorem 1] for weakly connected measurement graphs with independent relative measurements, under the assumption that either

(i) The covariance matrices of the measurements are exactly diagonal; or 
(ii) All measurements have exactly the same covariance matrix.

However, we would like our algorithm to be applicable to a wider case of relative noises, in particular to independent noises, with not necessarily diagonal or equal covariance matrices. Next we use results on block matrices [21], see Appendix A, to prove the convergence of the Jacobi algorithm for this more general case.

Theorem 2. Let the measurement graph $\mathcal{G}$ be weakly connected, $\left(\mathcal{G}_{\text {com }}\right.$ connected), $\Sigma_{\mathbf{z}_{1}}, \ldots, \Sigma_{\mathbf{z}_{m}}$ be the covariance matrices, not necessarily equal or diagonal, associated to $m$ independent $p$-dimensional measurements, and $\Sigma_{\mathbf{z}}$ be their associated block-diagonal covariance matrix as in eq. (1). Then, the spectral radius of $D^{-1} N$, with $D$ and $N$ as in eqs. (3)-(4), is less than 1, that is,

$$
\rho\left(D^{-1} N\right)<1
$$

Proof. In order to prove (8) we use the definitions and results in Appendix A. We first analyze the contents of $\Upsilon$ and show that $\Upsilon$ is of class $Z_{n-1}^{p}$ according to Definition 7 in Appendix A. Then, we use Lemma 8 and Theorem 9 to show that $\Upsilon$ is of class $M_{n-1}^{p}$ as in Definition 7. Finally, we show that $\Upsilon+\Upsilon^{T} \in M_{n-1}^{p}$ and use Theorem 10 to prove (8). Note that the subscript $n-1$ used in this proof instead of $n$ comes from the fact that $\Upsilon=\left[\Upsilon_{i j}\right]$, with $i, j \in \mathcal{V}^{a}$ and $\left|\mathcal{V}^{a}\right|=n-1$.

We first analyze the contents of the information matrix $\Upsilon$ given by eq. (3). Each block $\Upsilon_{i j}$ of the information matrix $\Upsilon$ is given by

$$
\Upsilon_{i i}=\sum_{j \in \mathcal{N}_{i}} \mathcal{B}_{i j} ; \quad \Upsilon_{i j}=-\mathcal{B}_{i j} \text { if } j \in \mathcal{N}_{i}, j \neq i ; \quad \Upsilon_{i j}=\mathbf{0} \text { if } j \notin \mathcal{N}_{i}, j \neq i ;
$$

for $i, j \in \mathcal{V}^{a}$, where $\mathcal{B}_{i j}$ is given by eq. (7). Note that $\mathcal{B}_{i j}$ is symmetric and that $\mathcal{B}_{i j} \succ \mathbf{0}^{2}$ and thus $-\mathcal{B}_{i j} \prec \mathbf{0}$ and symmetric. Therefore, matrix $\Upsilon$ is of class $Z_{n-1}^{p}$ according to Definition 7 .

\footnotetext{
${ }^{2} A \succ B(A \succeq B)$ indicates that matrix $A-B$ is positive-definite (positive-semidefinite). Equivalently, $\prec, \preceq$ are used for negative-definite and negative-semidefinite matrices.
} 
Now we focus on Lemma 8. We are interested in showing that, given any subset of agents $\mathcal{J} \subset \mathcal{V}^{a}$, there exists $i \in \mathcal{J}$ such that $\sum_{j \in \mathcal{J}} \Upsilon_{i j} \succ \mathbf{0}$. First we analyze the case $\mathcal{J}=\mathcal{V}^{a}$. Observe that $\Upsilon$ does not have rows or columns associated to the anchor agent $a$, i.e., $\Upsilon=\left[\Upsilon_{i j}\right]$ with $i, j \in \mathcal{V}^{a}$. On the other hand, for each agent $i$ that has the anchor $a$ as a neighbor, $a \in \mathcal{N}_{i}$, the block $\Upsilon_{i i}$ includes $\mathcal{B}_{i a}$. Therefore, $\sum_{j \in \mathcal{V}^{a}} \Upsilon_{i j} \succeq \mathbf{0}$ for all $i \in \mathcal{V}^{a}$, specifically

$$
\sum_{j \in \mathcal{V}^{a}} \Upsilon_{i j}=\mathbf{0} \text { if } a \notin \mathcal{N}_{i} \text {, and } \quad \sum_{j \in \mathcal{V}^{a}} \Upsilon_{i j}=\mathcal{B}_{i a} \succ \mathbf{0} \text {, when } a \in \mathcal{N}_{i} \text {. }
$$

Since $\mathcal{G}_{\text {com }}$ is connected, $a \in \mathcal{N}_{i}$ for at least one agent $i \in \mathcal{V}^{a}$. Now consider a proper subset $\mathcal{J} \varsubsetneqq \mathcal{V}^{a}$. Note that for each $i \in \mathcal{J} \varsubsetneqq \mathcal{V}^{a}$,

$$
\sum_{j \in \mathcal{J}} \Upsilon_{i j}=\mathbf{0} \text { if } \mathcal{N}_{i} \subseteq \mathcal{J} \text {, and } \quad \sum_{j \in \mathcal{J}} \Upsilon_{i j}=\sum_{j \in \mathcal{N}_{i} \backslash \mathcal{J}} \mathcal{B}_{i j} \succ \mathbf{0} \text {, otherwise. }
$$

Since $\mathcal{G}_{\text {com }}$ is connected, given any proper subset $\mathcal{J} \varsubsetneqq \mathcal{V}^{a}$ of agents, there is always an agent $i \in \mathcal{J}$ that has at least one neighbor outside $\mathcal{J}$ or that has the anchor $a$ as a neighbor, for which $\sum_{j \in \mathcal{J}} \Upsilon_{i j} \succ \mathbf{0}$. Therefore Lemma 8 holds, and by applying Theorem 9 taking $u_{2}, \ldots, u_{n}=1$ we conclude that matrix $\Upsilon \in M_{n-1}^{p}$. Since $\Upsilon$ is symmetric, then $\Upsilon+\Upsilon^{T} \in M_{n-1}^{p}$, and by [21, Theorem 4.7] we conclude that $\rho\left(D^{-1} N\right)<1$.

Corollary 3. Let $\mathcal{G}$ be weakly connected, equivalently $\mathcal{G}_{\text {com }}$ connected, $\Sigma_{\mathbf{z}_{1}}, \ldots, \Sigma_{\mathbf{z}_{m}}$ be the covariance matrices associated to $m$ independent $p$-dimensional measurements, and $\Sigma_{\mathbf{z}}$ be their associated block-diagonal covariance matrix as in eq. (1). Consider that each agent $i \in \mathcal{V}$ executes the Algorithm 1 to update its variable $\hat{\mathbf{x}}_{i}^{a}(t)$. Then, for all $i \in \mathcal{V}$,

$$
\lim _{t \rightarrow \infty} \hat{\mathbf{x}}_{i}^{a}(t)=\hat{\mathbf{x}}_{i}^{a}
$$

converges to the anchor-based centralized solution $\hat{\mathbf{x}}_{i}^{a}$ given by eq. (2).

\section{CENTROID ESTIMATION}

The accuracy of the estimated states $\hat{\mathbf{x}}_{\mathcal{V}}^{a}, \Sigma_{\hat{\mathbf{x}}_{\mathcal{V}}^{a}}$ in anchor-based approaches depends on the selected anchor $a$. Instead, we compute the states of the agents 
$\hat{\mathbf{x}}_{\mathcal{V}}^{c e n}, \Sigma_{\hat{\mathbf{x}}_{\mathcal{V}}^{c e n}}$ relative to the centroid given by the average of the states,

$$
\begin{aligned}
& \hat{\mathbf{x}}_{\mathcal{V}}^{c e n}=\left(\mathbf{I}-H_{c e n}\right) \hat{\mathbf{x}}_{\mathcal{V}}^{a}, \quad \Sigma_{\hat{\mathbf{x}}_{\mathcal{V}}^{c e n}}=\left(\mathbf{I}-H_{c e n}\right) \Sigma_{\hat{\mathbf{x}}_{\mathcal{V}}^{a}}\left(\mathbf{I}-H_{c e n}\right)^{T}, \\
& \text { where } H_{c e n}=\left(\mathbf{1}_{n} \otimes \mathbf{I}_{p}\right)\left(\mathbf{1}_{n} \otimes \mathbf{I}_{p}\right)^{T} / n .
\end{aligned}
$$

The interest of this representation is that the states of the agents $\hat{\mathbf{x}}_{\mathcal{V}}^{\text {cen }}, \Sigma_{\hat{\mathbf{x}}_{\mathcal{V}}^{\text {cen }}}$ with respect to the centroid are the same regardless of the anchor agent, i.e., the centroid solution is unique. Additionally, as the following result shows, it produces more accurate estimates than the ones provided by any anchor selection. We compare the block-traces ${ }^{3}$ blkTr of their covariance matrices [18].

Proposition 4. The covariance matrices of the centroid-based $\Sigma_{\hat{\mathbf{x}}_{\mathcal{V}}^{c e n}}$ and anchorbased $\Sigma_{\hat{\mathbf{x}}_{\mathcal{V}}^{a}}$ estimates satisfy, for all anchors $a \in \mathcal{V}$,

$$
\operatorname{blk} \operatorname{Tr}\left(\Sigma_{\hat{\mathbf{x}}_{\mathcal{V}}^{c e n}}\right) \preceq \operatorname{blk} \operatorname{Tr}\left(\Sigma_{\hat{\mathbf{x}}_{\mathcal{V}}^{a}}\right), \quad \operatorname{Tr}\left(\Sigma_{\hat{\mathbf{x}}_{\mathcal{V}}^{\text {cen }}}\right) \leq \operatorname{Tr}\left(\Sigma_{\hat{\mathbf{x}}_{\mathcal{V}}^{a}}\right) .
$$

Proof. Let $P_{i j}$ and $Q_{i j}$ be the $p \times p$ blocks of, respectively, the anchor and the centroid-based covariances, $\Sigma_{\hat{\mathbf{x}}_{\mathcal{V}}^{a}}=\left[P_{i j}\right], \Sigma_{\hat{\mathbf{x}}_{\mathcal{V}}^{c e n}}=\left[Q_{i j}\right]$ with $i, j \in \mathcal{V}$. The block-trace of the anchor-based covariance matrix is

$$
\operatorname{blk} \operatorname{Tr}\left(\Sigma_{\hat{\mathbf{x}}_{\mathcal{V}}^{a}}\right)=\sum_{i=1}^{n} P_{i i} .
$$

Considering eq. (13), each block in the main diagonal of the centroid-based $\Sigma_{\hat{\mathbf{x}}_{\mathcal{V}}^{\text {cen }}}$ covariance matrix, for all $i \in \mathcal{V}$, is given by

$$
Q_{i i}=P_{i i}-\frac{1}{n} \sum_{j=1}^{n}\left(P_{i j}+P_{j i}\right)+\frac{1}{n^{2}} \sum_{j=1}^{n} \sum_{j^{\prime}=1}^{n} P_{j j^{\prime}}
$$

and its block-trace $\operatorname{blk} \operatorname{Tr}\left(\sum_{\hat{\mathbf{x}}_{\mathcal{V}}^{\text {cen }}}\right)=\sum_{i=1}^{n} Q_{i i}=\sum_{i=1}^{n} P_{i i}-\sum_{i=1}^{n} \sum_{j=1}^{n} \frac{P_{i j}}{n}$ is

$$
\operatorname{blk} \operatorname{Tr}\left(\Sigma_{\hat{\mathbf{x}}_{\mathcal{V}}^{c e n}}\right)=\operatorname{blk} \operatorname{Tr}\left(\Sigma_{\hat{\mathbf{x}}_{\mathcal{V}}^{a}}\right)-\left(\mathbf{1}_{n} \otimes \mathbf{I}_{p}\right)^{T} \sum_{\hat{\mathbf{x}}_{\mathcal{V}}^{a}}\left(\mathbf{1}_{n} \otimes \mathbf{I}_{p}\right) / n
$$

Since $\Sigma_{\hat{\mathbf{x}}_{\mathcal{V}}^{a}}$ is symmetric and positive-semidefinite, then $\left(\mathbf{1}_{n} \otimes \mathbf{I}_{p}\right)^{T} \Sigma_{\hat{\mathbf{x}}_{\mathcal{V}}^{a}}\left(\mathbf{1}_{n} \otimes \mathbf{I}_{p}\right) \succeq$ $\mathbf{0}$, and thus $\operatorname{blk} \operatorname{Tr}\left(\sum_{\hat{\mathbf{x}}_{\mathcal{V}}^{c e n}}\right)-\operatorname{blk} \operatorname{Tr}\left(\sum_{\hat{\mathbf{x}}_{\mathcal{V}}^{a}}\right) \preceq \mathbf{0}$, as in eq. (14). Observe that the

\footnotetext{
${ }^{3}$ The block-trace of a matrix defined by blocks $P=\left[P_{i j}\right]$ with $i, j \in\{1, \ldots, n\}$ is the sum of its diagonal blocks, blk $\operatorname{Tr}(P)=\sum_{i=1}^{n} P_{i i}$
} 
trace of the block-trace of a matrix $A$ is equal to its trace, $\operatorname{Tr}(\operatorname{blk} \operatorname{Tr}(A))=\operatorname{Tr}(A)$. Since $\operatorname{blk} \operatorname{Tr}\left(\Sigma_{\hat{\mathbf{x}}_{\nu}^{c e n}}\right)-\operatorname{blk} \operatorname{Tr}\left(\Sigma_{\hat{\mathbf{x}}_{\mathcal{V}}^{a}}\right) \preceq \mathbf{0}$, the elements in the main diagonal of $\operatorname{blk} \operatorname{Tr}\left(\Sigma_{\hat{\mathbf{x}}_{\nu}^{e n}}\right)$ are smaller than or equal to the ones in the main diagonal of $\operatorname{blk} \operatorname{Tr}\left(\Sigma_{\hat{\mathbf{x}}_{v}^{a}}\right)$ so that

$$
\operatorname{Tr}\left(\sum_{\hat{\mathbf{x}}_{\mathcal{V}}^{c e n}}\right)=\operatorname{Tr}\left(\operatorname{blk} \operatorname{Tr}\left(\sum_{\hat{\mathbf{x}}_{\mathcal{V}}^{c e n}}\right)\right) \leq \operatorname{Tr}\left(\operatorname{blk} \operatorname{Tr}\left(\sum_{\hat{\mathbf{x}}_{\mathcal{V}}^{a}}\right)\right)=\operatorname{Tr}\left(\Sigma_{\hat{\mathbf{x}}_{\mathcal{V}}^{a}}\right)
$$

In particular, from eq. (17), $\operatorname{Tr}\left(\sum_{\hat{\mathbf{x}}_{\mathcal{V}}^{a}}\right)-\operatorname{Tr}\left(\sum_{\hat{\mathbf{x}}_{\mathcal{V}}^{\text {cen }}}\right)=\frac{1}{n} \sum_{i=1}^{n} \sum_{j=1}^{n} \operatorname{Tr}\left(P_{i j}\right)$. Note that the previous result holds when the anchor state $\hat{\mathbf{x}}_{a}^{a}$ is set to a general value, not necessarily $\mathbf{0}$. It also holds when there is more than one anchor. Consider that the first $k$ agents are anchors. In this case, matrix $\Sigma_{\hat{\mathbf{x}}_{\mathcal{V}}^{a}}=\left[P_{i j}\right]$ has its blocks $P_{i j}=\mathbf{0}$ for $i, j \in\{1, \ldots, k\}$, and eq. (17) gives $\operatorname{blk} \operatorname{Tr}\left(\Sigma_{\hat{\mathbf{x}}_{\mathcal{V}}^{\text {cen }}}\right)=$ $\operatorname{blk} \operatorname{Tr}\left(\sum_{\hat{\mathbf{x}}_{\mathcal{V}}^{a}}\right)-\sum_{i=k+1}^{n} \sum_{j=k+1}^{n} P_{i j} / n$, where $\sum_{i=k+1}^{n} \sum_{j=k+1}^{n} P_{i j} / n \succeq \mathbf{0}$.

We propose an algorithm that allows each agent $i \in \mathcal{V}$ to compute its state $\hat{\mathbf{x}}_{i}^{\text {cen }}$ with respect to the centroid in a distributed fashion, where $\hat{\mathbf{x}}_{\mathcal{V}}^{\text {cen }}=$ $\left(\left(\hat{\mathbf{x}}_{1}^{c e n}\right)^{T}, \ldots,\left(\hat{\mathbf{x}}_{n}^{c e n}\right)^{T}\right)^{T}$ is given in eq. (13). These states sum up to zero, $\hat{\mathbf{x}}_{1}^{c e n}+\cdots+\hat{\mathbf{x}}_{n}^{c e n}=\mathbf{0}$, since $\left(\mathbf{1}_{n} \otimes \mathbf{I}_{p}\right)\left(\mathbf{I}-H_{c e n}\right)=\mathbf{0}$, and for neighboring agents $i$ and $j$ satisfy $\hat{\mathbf{x}}_{i}^{c e n}=\hat{\mathbf{x}}_{j}^{c e n}-\hat{\mathbf{x}}_{j}^{a}+\hat{\mathbf{x}}_{i}^{a}$, where $\hat{\mathbf{x}}_{\mathcal{V}}^{a}=\left(\left(\hat{\mathbf{x}}_{1}^{a}\right)^{T}, \ldots,\left(\hat{\mathbf{x}}_{n}^{a}\right)^{T}\right)^{T}$. Thus, a straightforward solution would consist of firstly computing the anchorbased states of the agents $\hat{\mathbf{x}}_{\mathcal{V}}^{a}$, and in a second phase initializing the agents' variables so that they sum up to zero, $\hat{\mathbf{x}}_{i}^{\text {cen }}(0)=\mathbf{0}$, for $i \in \mathcal{V}$, and updating them at each step $t$ with an averaging algorithm that conserves the sum:

$$
\hat{\mathbf{x}}_{i}^{c e n}(t+1)=\sum_{j \in \mathcal{N}_{i} \cup\{i\}} \mathcal{W}_{i, j}\left(\hat{\mathbf{x}}_{j}^{c e n}(t)-\hat{\mathbf{x}}_{j}^{a}+\hat{\mathbf{x}}_{i}^{a}\right)
$$

for $i \in \mathcal{V}$, where $\mathcal{W}=\left[\mathcal{W}_{i, j}\right]$ is a doubly stochastic weight matrix such that $\mathcal{W}_{i, j}>0$ if $(i, j) \in \mathcal{E}$ and $\mathcal{W}_{i, j}=0$ when $j \notin \mathcal{N}_{i}$. Besides, $\mathcal{W}_{i, i} \in[\alpha, 1]$, $\mathcal{W}_{i, j} \in\{0\} \cup[\alpha, 1]$ for all $i, j \in \mathcal{V}$, for some $\alpha \in(0,1]$. More information about averaging algorithms can be found at [22-24]. The term $-\hat{\mathbf{x}}_{j}^{a}+\hat{\mathbf{x}}_{i}^{a}$ is the relative measurement $\mathbf{z}_{e}$ with $e=(j, i)$ for noise free scenarios, and the optimal or corrected measurement [11] $\hat{\mathbf{z}}_{e}$ for the noisy case, $\hat{\mathbf{z}}=\left(\mathcal{A} \otimes \mathbf{I}_{p}\right)^{T} \hat{\mathbf{x}}_{\mathcal{V}}^{a}$, with $\hat{\mathbf{z}}=\left(\left(\hat{\mathbf{z}}_{1}\right)^{T}, \ldots,\left(\hat{\mathbf{z}}_{m}\right)^{T}\right)^{T}$. In what follows we propose an algorithm where, at 
each iteration $t,(18)$ is executed not on the exact $\hat{\mathbf{x}}_{i}^{a}, \hat{\mathbf{x}}_{j}^{a}$, but on the most recent estimates $\hat{\mathbf{x}}_{i}^{a}(t), \hat{\mathbf{x}}_{j}^{a}(t)$ obtained with Algorithm 1 .

\section{DISTRIBUTED CENTROID ESTIMATION ALGORITHM}

Now we are ready to present the distributed algorithm for estimating the states of the agents relative to the centroid.

Algorithm 5. Let each agent $i \in \mathcal{V}$ have an estimate of its own state relative to the centroid, $\hat{\mathbf{x}}_{i}^{\text {cen }}(t) \in \mathbb{R}^{p}$, initialized at $t=0$ with $\hat{\mathbf{x}}_{i}^{\text {cen }}(0)=\mathbf{0}$. At each time step $t$, each agent $i \in \mathcal{V}$ updates $\hat{\mathbf{x}}_{i}^{\text {cen }}(t)$ with

$$
\hat{\mathbf{x}}_{i}^{c e n}(t+1)=\sum_{j \in \mathcal{N}_{i} \cup\{i\}} \mathcal{W}_{i, j}\left(\hat{\mathbf{x}}_{j}^{c e n}(t)+\hat{\mathbf{x}}_{i}^{a}(t)-\hat{\mathbf{x}}_{j}^{a}(t)\right),
$$

where $\hat{\mathbf{x}}_{i}^{a}(t), \hat{\mathbf{x}}_{j}^{a}(t)$ are the most recent estimates that agents $i$ and $j$ have at iteration $t$ of the variables in Algorithm 1 and $\mathcal{W}_{i, j}$ are the Metropolis weights of $\mathcal{G}_{\text {com }}$ as defined in [24].

Theorem 6. Let all the agents $i \in \mathcal{V}$ execute the Algorithm 5 and let $\mathcal{G}$ be weakly connected, equivalently $\mathcal{G}_{\mathrm{com}}$ connected. Then, the estimated states $\hat{\mathbf{x}}_{i}^{\text {cen }}(t)$ at each agent $i \in \mathcal{V}$ asymptotically converge to the state of $i$ relative to the centroid $\hat{\mathbf{x}}_{i}^{\text {cen }}$ given by eq. (13),

$$
\lim _{t \rightarrow \infty} \hat{\mathbf{x}}_{i}^{c e n}(t)=\hat{\mathbf{x}}_{i}^{c e n}
$$

Let $\mathbf{e}_{c e n}(t)=\left[\left(\hat{\mathbf{x}}_{1}^{c e n}(t)-\hat{\mathbf{x}}_{1}^{c e n}\right)^{T}, \ldots,\left(\hat{\mathbf{x}}_{n}^{c e n}(t)-\hat{\mathbf{x}}_{n}^{\text {cen }}\right)^{T}\right]^{T}$ be the error vector containing the estimation errors of the $n$ agents at iteration $t$. For fixed graphs $\mathcal{G}, \mathcal{G}_{\mathrm{com}}$ the norm of the error vector after $t$ iterations of Algorithm 5 satisfies

$$
\left\|\mathbf{e}_{c e n}(t)\right\|_{2} \leq \lambda_{\text {eff }}^{t}(\mathcal{W})\left\|\mathbf{e}_{c e n}(0)\right\|_{2}+2 p(n-1) \sigma_{J} \lambda_{\text {eff }}^{t}(\mathcal{W}) \sum_{k=1}^{t}\left(\frac{\rho(J)}{\lambda_{\text {eff }}(\mathcal{W})}\right)^{k}
$$

where $J$ is the Jacobi matrix $J=D^{-1} N$, with $D$ and $N$ computed as in eqs. (3)(4), $\sigma_{J}$ is a constant that depends on the initial Jacobi error and on $J . \mathcal{W}$ is the Metropolis weight matrix [24], and $\mathbf{e}_{\text {cen }}(0)$ is the initial error at $t=0$. 
Proof. First of all, we derive the expression for the convergence rate in eq. (21).

We express Algorithm 5 in terms of the error vectors associated to the centroid $\mathbf{e}_{c e n}(t)$ and the anchor-based $\mathbf{e}_{a}(t) \in \mathbb{R}^{(n-1) p}$ estimation methods (Algorithms 1 and 5), $\mathbf{e}_{c e n}(t)=\left[\left(\hat{\mathbf{x}}_{1}^{c e n}(t)\right)^{T}, \ldots,\left(\hat{\mathbf{x}}_{n}^{c e n}(t)\right)^{T}\right]^{T}-\hat{\mathbf{x}}_{\mathcal{V}}^{c e n}$, with $\hat{\mathbf{x}}_{\mathcal{V}}^{c e n}=$ $\left[\left(\hat{\mathbf{x}}_{1}^{c e n}\right)^{T}, \ldots,\left(\hat{\mathbf{x}}_{n}^{c e n}\right)^{T}\right]^{T}$ given by eq. (13), and $\tilde{\mathbf{e}}_{a}(t)=\left[\left(\hat{\mathbf{x}}_{2}^{a}(t)^{T}, \ldots, \hat{\mathbf{x}}_{n}^{a}(t)^{T}\right]^{T}-\right.$ $\hat{\mathbf{x}}_{\mathcal{V}^{a}}^{a}$, with $\hat{\mathbf{x}}_{\mathcal{V}^{a}}^{a}=\left[\left(\hat{\mathbf{x}}_{2}^{a}\right)^{T}, \ldots,\left(\hat{\mathbf{x}}_{n}^{a}\right)^{T}\right]^{T}$ given by eq. (2), where for simplicity we let the agent $i=1$ be the anchor $a$. We let $\mathbf{e}_{a}(t)$ be $\left(\mathbf{0}_{p}^{T}, \tilde{\mathbf{e}}_{a}(t)^{T}\right)^{T}$. Recall that $\sum_{j \in \mathcal{N}_{i} \cup\{i\}} \hat{\mathbf{x}}_{i}^{a}(t)=\hat{\mathbf{x}}_{i}^{a}(t)$ and that the estimated states relative to the centroid $\hat{\mathbf{x}}_{\mathcal{V}}^{c e n}$ are $\hat{\mathbf{x}}_{\mathcal{V}}^{c e n}=\left(\mathbf{I}-H_{\text {cen }}\right) \hat{\mathbf{x}}_{\mathcal{V}}^{a}$ as in eq. (13). Algorithm 5 becomes

$$
\mathbf{e}_{c e n}(t)=\left(\mathcal{W} \otimes \mathbf{I}_{p}\right) \mathbf{e}_{c e n}(t-1)+\left(\left(\mathbf{I}_{n}-\mathcal{W}\right) \otimes \mathbf{I}_{p}\right) \mathbf{e}_{a}(t-1)+P \hat{\mathbf{x}}_{\mathcal{V}}^{a},
$$

where the term $P$ that is multiplying $\hat{\mathbf{x}}_{\mathcal{V}}^{a}$ is

$$
P=\mathbf{I}-\left(\mathcal{W} \otimes \mathbf{I}_{p}\right)-\left(\mathbf{I}-\left(\mathcal{W} \otimes \mathbf{I}_{p}\right)\right)\left(\mathbf{I}-H_{c e n}\right)=\left(\mathbf{I}-\left(\mathcal{W} \otimes \mathbf{I}_{p}\right)\right) H_{c e n}
$$

We use the fact that $\left(\mathcal{W} \otimes \mathbf{I}_{p}\right) H_{c e n}=H_{c e n}$, and the previous expression gives $P=\mathbf{0}$ and eq. (22) becomes

$$
\begin{aligned}
\mathbf{e}_{c e n}(t) & =\left(\mathcal{W} \otimes \mathbf{I}_{p}\right) \mathbf{e}_{c e n}(t-1)+\left(\left(\mathbf{I}_{n}-\mathcal{W}\right) \otimes \mathbf{I}_{p}\right) \mathbf{e}_{a}(t-1)= \\
& =\left(\mathcal{W} \otimes \mathbf{I}_{p}\right)^{t} \mathbf{e}_{c e n}(0)+\sum_{k=0}^{t-1}\left(\mathcal{W} \otimes \mathbf{I}_{p}\right)^{t-k-1}\left((\mathbf{I}-\mathcal{W}) \otimes \mathbf{I}_{p}\right) \mathbf{e}_{a}(k)
\end{aligned}
$$

Then, the norm of the error $\mathbf{e}_{c e n}(t)$ satisfies

$$
\left\|\mathbf{e}_{c e n}(t)\right\|_{2} \leq \lambda_{\text {eff }}^{t}(\mathcal{W})\left\|\mathbf{e}_{c e n}(0)\right\|_{2}+2 \sum_{k=0}^{t-1} \lambda_{\text {eff }}^{t-k-1}(\mathcal{W})\left\|\mathbf{e}_{a}(k)\right\|_{2},
$$

where we have used the fact that $\left\|\left((\mathcal{W}-\mathbf{I}) \otimes \mathbf{I}_{p}\right)\right\|_{2} \leq 2$ since $\mathcal{W}$ is the Metropolis weight matrix.

We analyze now the norm of error $\left\|\mathbf{e}_{a}(t)\right\|_{2}$, which is related to the error vector of the Jacobi algorithm $\tilde{\mathbf{e}}_{a}(t) \in \mathbb{R}^{(n-1) p}$ by $\mathbf{e}_{a}(t)=\left(\mathbf{0}_{p}^{T}, \tilde{\mathbf{e}}_{a}^{T}(t)\right)^{T}$. Let $J$ be the Jacobi matrix, and $V_{J}=\left[\mathbf{v}_{p+1}(J), \ldots, \mathbf{v}_{n p}(J)\right]$ and $\lambda_{J}=\operatorname{Diag}\left(\lambda_{p+1}(J), \ldots, \lambda_{n p}(J)\right)$ be its associated eigenvectors and eigenvalues so that $J=V_{J} \lambda_{J} V_{J}^{-1}$, and $\left\|\mathbf{v}_{i}(J)\right\|_{2}=1$. The error vector $\tilde{\mathbf{e}}_{a}(t)$ evolves according to

$$
\tilde{\mathbf{e}}_{a}(t)=J \tilde{\mathbf{e}}_{a}(t-1)=J^{t} \tilde{\mathbf{e}}_{a}(0) .
$$


For each initial error vector $\tilde{\mathbf{e}}_{a}(0)$ there exist $\sigma_{p+1}, \ldots, \sigma_{n p}$ such that

$$
\tilde{\mathbf{e}}_{a}(0)=\sum_{i=p+1}^{n p} \sigma_{i} \mathbf{v}_{i}(J)
$$

and then the error vector $\tilde{\mathbf{e}}_{a}(t)$ after $t$ iterations of the Jacobi algorithm given by eq. (26) can be expressed as

$$
\tilde{\mathbf{e}}_{a}(t)=V_{J} \lambda_{J}^{t} V_{J}^{-1} V_{J}\left[\sigma_{p+1}, \ldots, \sigma_{n p}\right]^{T}=\sum_{i=p+1}^{n p} \sigma_{i} \mathbf{v}_{i}(J) \lambda_{i}^{t}(J) .
$$

Let $\sigma_{J}=\max _{i=p+1}^{n p}\left|\sigma_{i}\right|$, and $\rho(J)=\max _{i=p+1}^{n p}\left|\lambda_{i}(J)\right|$. For all $t \geq 0$, the norm of the error vector $\left\|\tilde{\mathbf{e}}_{a}(t)\right\|_{2}$ satisfies

$$
\left\|\mathbf{e}_{a}(t)\right\|_{2}=\left\|\tilde{\mathbf{e}}_{a}(t)\right\|_{2} \leq p(n-1) \sigma_{J} \rho^{t}(J) .
$$

Linking this with eq. (25) gives that the convergence rate is

$$
\left\|\mathbf{e}_{c e n}(t)\right\|_{2} \leq \lambda_{\text {eff }}^{t}(\mathcal{W})\left\|\mathbf{e}_{c e n}(0)\right\|_{2}+2 p(n-1) \sigma_{J} \sum_{k=0}^{t-1} \lambda_{\text {eff }}^{t-k-1}(\mathcal{W}) \rho^{k}(J),
$$

as in eq. (21).

Now we prove the asymptotical convergence to the centroid (20). If both the Jacobi and the general algorithm have the same convergence rate, $\rho(J)=$ $\lambda_{\text {eff }}(\mathcal{W})$, then eq. $(28)$ gives

$$
\left\|\mathbf{e}_{c e n}(t)\right\|_{2} \leq \lambda_{\text {eff }}^{t}(\mathcal{W})\left\|\mathbf{e}_{c e n}(0)\right\|_{2}+2 p(n-1) \sigma_{J} \lambda_{\text {eff }}^{t-1}(\mathcal{W}) t
$$

whereas for $\rho(J) \neq \lambda_{\text {eff }}(\mathcal{W})$, it gives

$$
\left\|\mathbf{e}_{c e n}(t)\right\|_{2} \leq \lambda_{\text {eff }}^{t}(\mathcal{W})\left\|\mathbf{e}_{c e n}(0)\right\|_{2}+\frac{2 p(n-1) \sigma_{J}}{\rho(J)-\lambda_{\text {eff }}(\mathcal{W})}\left(\rho^{t}(J)-\lambda_{\text {eff }}^{t}(\mathcal{W})\right) .
$$

Note that $\lambda_{\text {eff }}(\mathcal{W})<1$ for connected graphs $\mathcal{G}_{\text {com. }}$. Then, the term $\lambda_{\text {eff }}^{t}(\mathcal{W})\left\|\mathbf{e}_{c e n}(0)\right\|_{2}$ in eqs. (29) and (30) exponentially tends to zero as $t \rightarrow \infty$ regardless of the initial error $\mathbf{e}_{c e n}(0)$. For the case $\rho(J)=\lambda_{\text {eff }}(\mathcal{W})$, the term $\lambda_{\text {eff }}^{t}(\mathcal{W}) t$ in eq. $(29)$ is decreasing for $t \geq \frac{\lambda_{\text {eff }}(\mathcal{W})}{1-\lambda_{\text {eff }}(\mathcal{W})}$ and thus it tends to zero as $t \rightarrow \infty$. For $\rho(J) \neq \lambda_{\text {eff }}(\mathcal{W})$, the term $\left(\rho^{t}(J)-\lambda_{\text {eff }}^{t}(\mathcal{W})\right)$ in eq. (30) asymptotically tends to zero since $\lambda_{\text {eff }}(\mathcal{W})$ is less than 1 , and as stated by Theorem $2, \rho(J)<1$. Therefore, $\lim _{t \rightarrow \infty}\left\|\mathbf{e}_{c e n}(t)\right\|_{2}=0$, where $\left\|\mathbf{e}_{c e n}(t)\right\|_{2}=0$ iff $\mathbf{e}_{c e n}(t)=0$, what concludes the proof. 
We finish this section with a brief explanation about the information measured and communicated by the agents during the execution of the algorithms presented so far. Each agent $i$ measures the relative position $\mathbf{z}_{e}, \Sigma_{\mathbf{z}_{e}}$, of its outneighbors $e=(i, j)$ in $G$ during an initial stage. During the general iterations of Algorithm 5 (centroid estimation) agent $i$ communicates the following pieces of information to its neighbors $\mathcal{N}_{i}: \hat{\mathbf{x}}_{i}^{a}(t)$ and $\hat{\mathbf{x}}_{i}^{c e n}(t)$.

In addition, agents execute a method to discover the graphs $\mathcal{G}$ and $\mathcal{G}_{\text {com }}$, to compute the Metropolis weights $\mathcal{W}_{i, j}$, and to inform of their relative measurements $\mathbf{z}_{e}, \Sigma_{\mathbf{z}_{e}}$ to their neighbors. It roughly consists of each agent $i$ sending messages to all agents $j$ in communication range, including its measurement $\mathbf{z}_{e}, \Sigma_{\mathbf{z}_{e}}$, with $e=(i, j)$, if available. Agent $i$ erases $(i, j)$ links from $\mathcal{G}$ if $j$ is not in communication range, and $\{i, j\}$ links from $\mathcal{G}_{\text {comm }}$ if no $\mathbf{z}_{e}$ measurements between $i$ and $j$ exist. Finally, each agent $i$ counts its remaining neighbors (its degree), informs its neighbors of its degree, and computes the Metropolis weights $\mathcal{W}_{i, j}$, and matrices $\mathcal{B}_{i j}, M_{i}$ in eqs. (6)-(7). This method is executed only once, immediately after the sensing stage, for graphs $\mathcal{G}, \mathcal{G}_{\text {com }}$ with fixed topology, as the ones considered in this paper. If the graphs were switching, this process would need to be executed before each iteration of Algorithm 5.

\section{SIMULATIONS}

We study the performance of the presented algorithm in a planar multiagent localization scenario (Fig. 1) with $n=20$ agents (circles) that get noisy measurements (crosses and ellipses) of the position of agents which are closer than 4 meters. Each agent $i \in \mathcal{V}$ is used as an anchor and its covariance matrix $\Sigma_{\hat{\mathbf{x}}_{\mathcal{V}}^{i}}$ is computed. The eigenvalues of the block-traces $\operatorname{blk} \operatorname{Tr}\left(\Sigma_{\hat{\mathbf{x}}_{\mathcal{V}}^{i}}\right)$ of their covariance matrices are depicted in Fig. 2 (a) (crosses). For all the possible anchors $i \in \mathcal{V}$, the eigenvalues associated to the anchor-based covariance matrices $\operatorname{blk} \operatorname{Tr}\left(\Sigma_{\hat{\mathbf{x}}_{V}^{i}}\right)$ are larger (more uncertain) than the ones associated to the centroid-based covariance matrix $\operatorname{blk} \operatorname{Tr}\left(\sum_{\hat{\mathbf{x}}_{\mathcal{V}}^{c e n}}\right)$ (circle). Agents $R 3$ and $R 12$ which produce respectively the most and the least precise anchor-based results, 


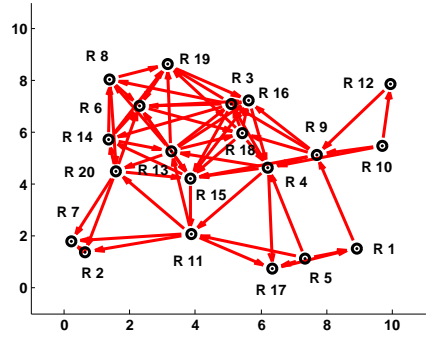

(a) Ground truth scenario

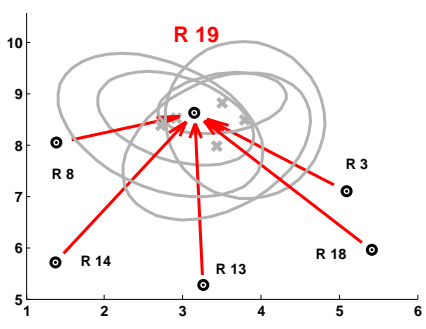

(b) Measurements of $R 19$ 's position

Figure 1: (a) 20 agents (circles) are placed randomly in a region of $10 \times 10$ meters. (b) Each agent $i$ measures $\mathbf{z}_{e}$ (crosses and ellipses) the relative position of its out-neighbors $j$ (arrows), with $e=(i, j)$, being the measurement noise proportional to the distance to $j$.

and agent $R 1$ which is conventionally used as the anchor (in blue) are studied in detail. Fig. 2 (b) shows the estimated states relative to the centroid $\hat{\mathbf{x}}_{\mathcal{V}}^{\text {cen }}, \Sigma_{\hat{\mathbf{x}}_{\mathcal{V}}^{\text {cen }}}$ (black crosses and ellipses) and the anchor $a=R 1, \hat{\mathbf{x}}_{\mathcal{V}}^{a}, \Sigma_{\hat{\mathbf{x}}_{\mathcal{V}}^{a}}$ (blue crosses and ellipses) compared with the ground-truth positions of the agents (red crosses). The ground-truth position of the centroid is marked with a black ' $x$ '. The errors and covariances associated to the centroid-based estimates (black) are in general smaller than the ones obtained by using $R 1$ as the anchor (blue).

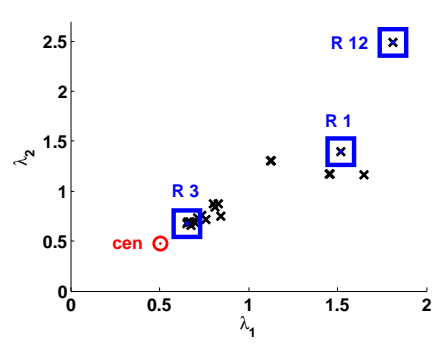

(a) Eigenvalues of $\operatorname{blk} \operatorname{Tr}\left(\Sigma_{\hat{\mathbf{x}}_{\mathcal{V}}^{i}}\right)$

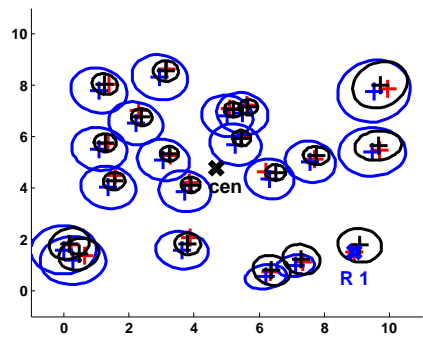

(b) Estimated positions

Figure 2: (a) The eigenvalues of $\operatorname{blk} \operatorname{Tr}\left(\Sigma_{\hat{\mathbf{x}}_{V}^{i}}\right)$ when each agent $i \in \mathcal{V}$ is taken as the anchor (crosses) are always greater than the ones of the centroid-based $\operatorname{blk} \operatorname{Tr}\left(\Sigma_{\hat{\mathbf{x}}_{\mathcal{V}}^{\text {cen }}}\right)$ representation (circle). (b) The positions estimated relative to the centroid (black crosses and ellipses) are in general more accurate and closer to the ground-truth (red crosses) than the ones estimated by using $R 1$ as the anchor (blue crosses and ellipses).

We analyze the states estimated by the $n$ agents along 1000 iterations of 
the proposed algorithm (Fig. 3). Agents initialize their states $\hat{\mathbf{x}}_{i}^{a}(t), \hat{\mathbf{x}}_{i}^{c e n}(t)$ with zeros and execute Algorithms 1 and 5. We generate specific noises as the ones in Fig. 1 for 100 different samples. For each of them, we record the norm of the error vector containing the difference between the estimates at the $n$ agents and the ground-truth positions at each iteration $t$. In Fig. 3 (a) we show the results of Algorithm 1 when each agent $i \in \mathcal{V}$ is used as the anchor (gray lines). The special cases that the anchor is $R 1, R 3$ and $R 12$ are displayed in blue. The black line is the asymptotic error reached with the centroid-based estimation method. As it can be seen, the errors reached with the anchor-based solutions are greater than the ones associated to the centroid. This is even more evident in Fig. 3 (b), which shows the last 100 iterations in Fig. 3 (a). In Fig. 3 (c) we show the equivalent errors for the centroid-based estimation algorithm (Algorithm 5), using all the possible anchors for Algorithm 1. The anchor choice affects to the convergence speed of Algorithm 5. However, the final accuracy is independent on the selected anchor, and in all cases the error estimates (gray lines) converge to the asymptotic centroid error (black line).

\section{CONCLUSIONS}

We have presented an algorithm to estimate the states of a set of agents from noisy measurements of the relative states of neighbors. The interest of the presented algorithm is that the states are estimated relative to the centroid, instead of relative to an anchor. Our algorithm consists of the simultaneous computation of the centroid and of the anchor-based states. The anchor-based estimation is carried out with the Jacobi algorithm and we give theoretical and experimental proofs of convergence for general block diagonal covariance matrices. The centroid-based approach relies on distributed averaging of the most recent anchor-based estimates. We give proofs of the convergence of the

whole procedure and we show that the centroid-based estimates are more precise than any anchor-based solution. 


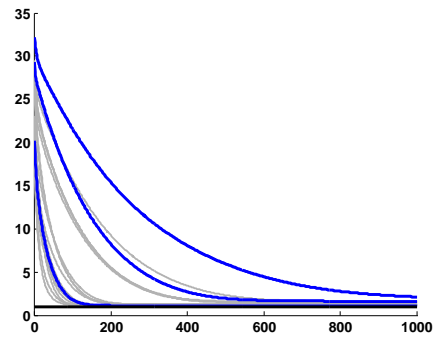

(a) Anchor-based errors

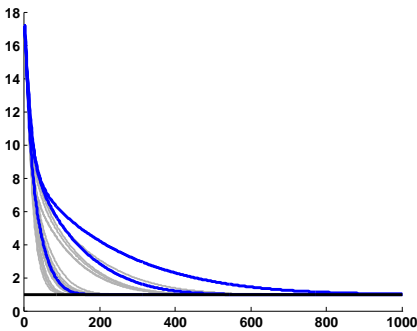

(c) Centroid-based errors

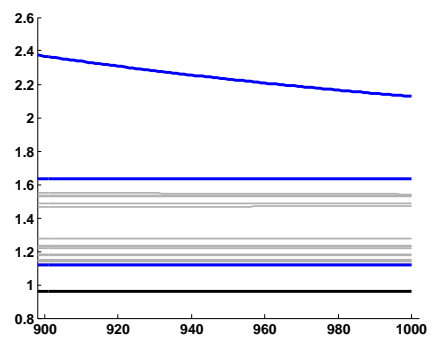

(b) Last 100 iterations of (a)

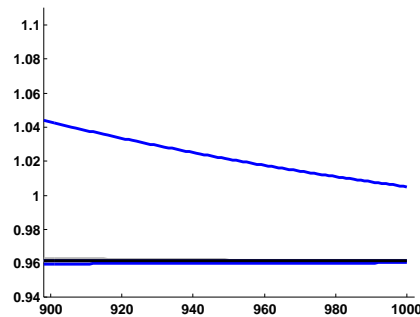

(d) Last 100 iterations of (c)

Figure 3: Norm of the error with the difference between the estimates and the ground truth. (a)-(b) Results of Algorithm 1 when each node $i \in \mathcal{V}$ is used as the anchor (gray lines). The special cases that the anchor is $R 1, R 3$ and $R 12$ are shown in blue. The black line is the asymptotic error reached with the centroid-based estimation. (c)-(d) Results of the centroid-based estimation algorithm (Algorithm 5) using all the possible anchors.

\section{ACKNOWLEDGMENT}

This work was supported by Spanish Ministerio de Ciencia e Innovacion DPI2009-08126 project, and Italian Ministry of University and Research (MIUR) MEMONET National Research Project, and grants MEC BES-2007-14772, PRIN 20087W5P2K, and PRIN MEMONET.

\section{Appendix A. $Z_{N}^{P}$ AND $M_{N}^{P}$ MATRICES DEFINED BY BLOCKS}

The next properties are provided in [21] for matrices $A \in \mathbb{R}^{n p \times n p}$ defined by blocks, being each block $A_{i j}$ a $p \times p$ matrix, for all $i, j \in\{1, \ldots, n\}$.

Definition 7. [21] Matrix $A$ is of class $Z_{n}^{p}$ if $A_{i j}$ is symmetric for all $i, j \in$ $\{1, \ldots, n\}$ and $A_{i j} \preceq \mathbf{0}$ for all $i, j \in\{1, \ldots, n\}, j \neq i$. It is of class $\hat{Z}_{n}^{p}$ if $A \in Z_{n}^{p}$ 
and $A_{i i} \succ \mathbf{0}$ for all $i \in\{1, \ldots, n\}$, and of class $M_{n}^{p}$ if $A \in \hat{Z}_{n}^{p}$ and there exist scalars $u_{1}, \ldots, u_{n}>0$ such that $\sum_{j=1}^{n} u_{j} A_{i j} \succ \mathbf{0}$ for all $i \in\{1, \ldots, n\}$.

Lemma 8. [21, Lemma 3.8] Let $A \in Z_{n}^{p}$ and assume that $\forall \mathcal{J} \subset\{1, \ldots, n\}$ there exists $i \in \mathcal{J}$ such that $\sum_{j \in \mathcal{J}} A_{i j} \succ \mathbf{0}$. Then, there exists a permutation $\pi$ such that $\sum_{j \geq i} A_{\pi(i), \pi(j)} \succ \mathbf{0}$, for all $i \in\{1, \ldots, n\}$.

Theorem 9. [21, Theorem 3.11] Let $A \in Z_{n}^{p}, u_{1}, \ldots, u_{n}>0$, and $\sum_{j=1}^{n} A_{i j} u_{j} \succeq$ 0 for all $i \in\{1, \ldots, n\}$. Assume that there exists a permutation $\pi$ of $\{1, \ldots, n\}$ such that $\sum_{j \geq i} A_{\pi(i), \pi(j)} u_{\pi j} \succ \mathbf{0}$ for all $i \in\{1, \ldots, n\}$. Then, $A \in M_{n}^{p}$.

Theorem 10. [21, Theorem 4.7] Let $A+A^{T} \in M_{n}^{p}, D=\operatorname{blkDiag}\left(A_{11}, \ldots, A_{n n}\right)$, and $A=D-N$. Then $\rho\left(D^{-1} N\right)<1$.

\section{REFERENCES}

[1] B.S.Y.Rao, H.F.Durrant-Whyte, J.A.Sheen, A fully decentralized multisensor system for tracking and surveillance, International Journal of Robotics Research 12 (1) (1993) 20-44.

[2] F.Chen, W.Ren, Y.Cao, Surrounding control in cooperative agent networks, Systems \& Control Letters 59 (11) (2010) 704-712.

[3] A.Sarlette, R.Sepulchre, N.E.Leonard, Autonomous rigid body attitude synchronization, Automatica 45 (2) (2008) 572-577.

[4] J.Cortes, Global and robust formation-shape stabilization of relative sensing networks, Automatica 45 (12) (2009) 2754 - 2762.

[5] B.D.O.Anderson, I.Shames, G.Mao, B.Fidan, Formal theory of noisy sensor network localization, SIAM Journal on Discrete Mathematics 24 (2) (2010) 684-698.

[6] G.Calafiore, L.Carlone, M.Wei, A distributed gauss-newton approach for range-based localization of multi agent formations, in: IEEE Multi-Conf. on Systems and Control, Yokohama, Japan, 2010, pp. 1152-1157. 
[7] A.Savvides, W.L.Garber, R.L.Moses, M.B.Srivastava, An analysis of error inducing parameters in multihop sensor node localization, IEEE Transactions on Mobile Computing 4 (6) (2005) 567-577.

[8] C.Sagues, A.C.Murillo, J.J.Guerrero, T.Goedemé, T.Tuytelaars, L. Gool, Localization with omnidirectional images using the 1D radial trifocal tensor, in: IEEE Int. Conf. on Robotics and Automation, Orlando, USA, 2006, pp. $551-556$.

[9] N.Trawny, X.S.Zhou, K.X.Zhou, S.I.Roumeliotis, Inter-robot transformations in 3-d, IEEE Transactions on Robotics 26 (2) (2010) 226-243.

[10] P.Barooah, J.Hespanha, Distributed estimation from relative measurements in sensor networks, in: Int. Conf. on Intelligent Sensing and Information Processing, Chennai, India, 2005, pp. 88-93.

[11] W.J.Russell, D.Klein, J.P.Hespanha, Optimal estimation on the graph cycle space, in: American Control Conference, Baltimore, USA, 2010, pp. 19181924.

[12] G.Calafiore, F.Abrate, Distributed linear estimation over sensor networks, International Journal of Control 82 (5) (2009) 868-882.

[13] M.Franceschelli, A.Gasparri, On agreement problems with gossip algorithms in absence of common reference frames, in: IEEE Int. Conf. on Robotics and Automation, Anchorage, USA, 2010, pp. 4481-4486.

[14] J.Knuth, P.Barooah, Distributed collaborative localization of multiple vehicles from relative pose measurements, in: Allerton Conf. on Communications, Control and Computing, Urbana-Champaign, USA, 2009, pp. $314-321$.

[15] M.Ji, M.Egerstedt, Distributed coordination control of multiagent systems while preserving connectedness, IEEE Transactions on Robotics 23 (4) (2007) 693-703. 
[16] G.Lafferriere, A.Williams, J.Caughman, J.J.P.Veerman, Decentralized control of vehicle formations, Systems \& Control Letters 54 (9) (2005) 899-910.

[17] J.A.Fax, R.M.Murray, Information flow and cooperative control of vehicle formations, IEEE Transactions on Automatic Control 49 (9) (2004) 14651476.

[18] P.Barooah, J.Hespanha, Error scaling laws for linear optimal estimation from relative measurements, IEEE Transactions on Information Theory 55 (12) (2009) 5661-5673.

[19] D.P.Bertsekas, J.N.Tsitsiklis, Parallel and Distributed Computation: Numerical Methods, Athena Scientific, 1997.

[20] P.Barooah, J.Hespanha, Estimation on graphs from relative measurements, IEEE Control Systems Magazine 27 (4) (2007) 57-74.

[21] L.Elsner, V.Mehrmann, Convergence of block iterative methods for linear systems arising in the numerical solution of Euler equations, Numerische Mathematik 59 (1) (1991) 541-559.

[22] W.Ren, R.W.Beard, Distributed Consensus in Multi-vehicle Cooperative Control, Communications and Control Engineering, Springer Verlag, London, 2008.

[23] F.Bullo, J.Cortes, S.Martinez, Distributed Control of Robotic Networks, Applied Mathematics Series, Princeton University Press, 2009, electronically available at http://coordinationbook.info.

[24] L.Xiao, S.Boyd, S.Lall, A scheme for robust distributed sensor fusion based on average consensus, in: Symposium on Information Processing of Sensor Networks (IPSN), Los Angeles, CA, 2005, pp. 63-70. 